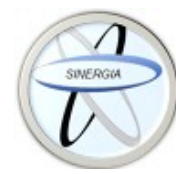

Revista Médica Sinergia

Vol. 6, Núm. 7, julio 2021, e687

\title{
Generalidades sobre cardiomiopatía periparto
} Overview of peripartum cardiomyopathy

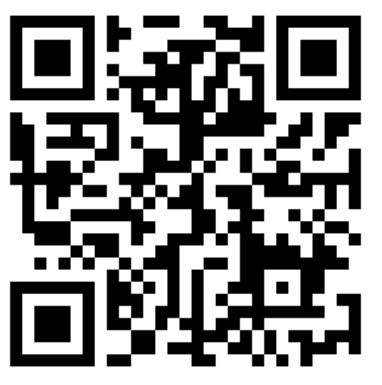

Recibido 19/04/2021
${ }^{1}$ Dr. Juan Carlos Garnier Fernández Investigador independiente, San José, Costa Rica

(iD) https://orcid.org/0000-0002-5716-4562

${ }^{2}$ Dra. Gloriana Pizarro Alvarado Investigadora independiente, Heredia, Costa Rica

(i) https://orcid.org/0000-0002-9178-2751

${ }^{3}$ Dr. Roberto Orozco García Investigador independiente, Alajuela, Costa Rica

https://orcid.org/0000-0003-4641-5001
Aceptado
$20 / 05 / 2021$

\section{RESUMEN}

La cardiomiopatía periparto se define como: una falla cardiaca aguda secundaria a disfunción sistólica, que se presenta hacia el final del embarazo o en los primeros cinco meses después del parto. Tiene una incidencia de 1 en 1,000 a 1 en 4,000 nacimientos.

El factor de riesgo más importante es ser de descendencia africana, otros factores son la edad materna avanzada, multiparidad y los trastornos hipertensivos. La fisiopatología sigue siendo desconocida, y se han propuesto como los causantes de la enfermedad los procesos autoinmunes, factores inflamatorios, infecciones agudas, malnutrición, alteraciones hormonales, apoptosis celular, disfunción y estrés oxidativo endotelial.

La clínica es variada y presentan fatiga, disnea de esfuerzo, disnea paroxística nocturna, tos, ortopnea, edema con fóvea, palpitaciones, dolor torácico, taquipnea, taquicardia, presión venosa yugular elevada, estertores pulmonares, ritmo de galope y ascitis. El diagnóstico es de exclusión, y se debe solicitar un ecocardiograma, electrocardiograma, medición de péptidos natriuréticos (BNP y NT pro-BNP), y radiografía de tórax.

El tratamiento es similar al de otras causas de insuficiencia cardíaca e incluye mantener una adecuada oxigenación, restricción de líquidos y sal, frenar la progresión de la disfunción del ventrículo izquierdo. Los fármacos utilizados son diuréticos, vasodilatadores, inotrópicos, Inhibidores de la enzima convertidora de angiotensina, inhibidores de los receptores II de angiotensina, beta bloqueadores, digoxina, espironolactona y anticoagulación con heparina. La mortalidad es aproximadamente del $4 \%$. 


\title{
PALABRAS CLAVE: cardiomiopatía periparto; embarazo; diagnostico; manejo;
} biomarcadores; falla cardiaca aguda; etnia.

\begin{abstract}
Peripartum cardiomyopathy is defined as: acute heart failure secondary to systolic dysfunction, occurring towards the end of pregnancy or in the first five months after delivery. It has an incidence of 1 in 1,000 to 1 in 4,000 births.

The most important risk factor is being of African descendence; other factors are advanced maternal age, multiparity and hypertensive disorders. The pathophysiology remains unknown, and autoimmune processes, inflammatory factors, acute infections, malnutrition, hormonal alterations, cellular apoptosis, endothelial dysfunction and oxidative stress have been proposed as the causes of the disease.

The clinical manifestations are varied and include fatigue, exertional dyspnea, paroxysmal nocturnal dyspnea, cough, orthopnea, edema with pitting, palpitations, chest pain, tachypnea, tachycardia, elevated jugular venous pressure, pulmonary rales, gallop rhythm and ascites. The diagnosis is of exclusion, and an echocardiogram, electrocardiogram, measurement of natriuretic peptides (BNP and NT pro-BNP), and chest X-ray should be requested.

Treatment is like other causes of heart failure and includes maintaining adequate oxygenation, fluid and salt restriction, and slowing the progression of left ventricular dysfunction. The drugs used are diuretics, vasodilators, inotropics, angiotensin converting enzyme inhibitors, angiotensin receptor II inhibitors, beta-blockers, digoxin, spironolactone and anticoagulation with heparin. Mortality is approximately $4 \%$.
\end{abstract}

KEYWORDS: peripartum cardiomyopathy; pregnancy; diagnosis; treatment; biomarker; acute heart failure; ethnicity.

\footnotetext{
${ }^{1}$ Médico general, graduado de la Universidad de Iberoamérica (UNIBE). Cód. MED16727. Correo: juankgarnier@gmail.com

${ }^{2}$ Médica general, graduada de la Universidad de Iberoamérica (UNIBE). Cód. MED16722. Correo: gloripa17@gmail.com

${ }^{3}$ Médico general, graduado de la Universidad de Iberoamérica (UNIBE). Cód. MED16746. Correo: rob.orozco.garcia@gmail.com
}

\section{INTRODUCCIÓN}

La cardiomiopatía periparto es una rara enfermedad asociada con el embarazo, la cual fue descrita por primera vez hace más de 150 años (1). La cual se define como: una cardiomiopatía idiopática asociada a falla cardiaca aguda secundaria a disfunción sistólica, que se presenta hacia el final del embarazo o en los primeros cinco meses después del parto, donde no se encuentra ninguna otra etiología cardiaca posible $(2,3)$. Se considera un diagnóstico de exclusión, por lo que requiere de gran sospecha clínica para una adecuada captación temprana y manejo oportuno, para prevenir sus posibles complicaciones. Tiene una prevalencia a nivel mundial, con una incidencia de 1 en 1,000-4,000 nacimientos en Estados Unidos, hasta 1 en 100 nacimientos en Nigeria y 1 en 300 nacimientos en Haití (2). Con una mortalidad importante, con un rango de 3$40 \%$ dependiendo de la localización geográfica (4) además, representa el $5 \%$ de los trasplantes cardiaco que se realizan en pacientes femeninas en Estados Unidos (5). Las complicaciones en su mayoría se asocian en paciente con predisposición genética, factores de riesgo y la frecuente captación tardía que presenta por su clínica 
similar a los cambios normales del embarazo. Por lo que, al tratarse de una enfermedad con una prevalencia creciente y una mortalidad nada despreciable, las revisiones bibliográficas permiten valorar la bibliografía más reciente sobre el tema, con el objetivo de mantener actualizado a los profesionales sobre el diagnóstico temprano y manejo integral de la patología.

\section{MÉTODO}

Al elaborar este artículo, la bibliografía fue tomada de diferentes bases de datos como Pubmed, Cochrane y Trip Medical Data Base, todas publicadas con 5 años o menos de antigüedad. Los términos que se utilizaron fueron "cardiomiopatía", "periparto", "embarazo", "biomarcadores", "diagnostico", "tratamiento", Se realizó la revisión con la finalidad de obtener la información más actualizada en el tema, la bibliografía utilizada fue en idioma inglés y español.

\section{DEFINICIÓN}

La cardiomiopatía periparto se define como: una falla cardiaca aguda secundaria a disfunción sistólica, que se presenta hacia el final del embarazo o en los primeros cinco meses después del parto, donde no se encuentra ninguna otra etiología cardiaca posible, ni existe enfermedad cardiaca estructural conocida, debe presentar signos clínicos de falla cardiaca aguda, así como, criterios ecocardiográficos que indiquen una disminución en la fracción de eyección del ventrículo izquierdo $\leq 45 \%$, puede o no haber dilatación ventricular $(1,2)$. Es de gran importancia recalcar que es un diagnóstico de exclusión, esto debido a que no existen pruebas específicas para confirmar el diagnóstico (6).

\section{EPIDEMIOLOGÍA}

La cardiomiopatía periparto tiene prevalencia a nivel mundial, con una incidencia que varía según la localización geográfica, en Estados Unidos se presenta con una incidencia de 1 en 1,000 a 1 en 4,000 nacimientos, la cual según estudios es similar en Europa y Asia $(2,4,5)$. Sin embargo, existen puntos geográficos donde la incidencia es considerablemente mayor, específicamente la población de Haití y Nigeria, los cuales presentan 1 en 300 nacimientos y 1 en 100 nacimientos respectivamente (2) lo que habla de una marcada diferencia según etnia.

Existe franca predisposición de mujeres de descendencia africana en relación con la patología, ya que, en estas, la enfermedad se presenta a menor edad, en un periodo posparto más avanzado y con fracciones de eyecciones del ventrículo izquierdo menores, usualmente menor a $30 \%$ (6) esto en comparación con mujeres sin descendencia africana.

Más del $90 \%$ de los casos se presentan en el periodo posparto, la mayoría de las mujeres caucásicas presentan la enfermedad en la primera semana posparto, a diferencia de las mujeres de descendencia africana, las cuales la presenta significativamente después (5).

Las tasas de incidencia en países desarrollados han ido en aumento durante los últimos años, esto relacionado con una mayor captación de la enfermedad asociado a facilidad de acceso a métodos diagnósticos como el ecocardiograma, pero también por factores de riesgo documentados (4).

\section{FACTORES DE RIESGO}

Diferentes factores de riesgo implicados con el desarrollo de la cardiomiopatía periparto han sido descritos, el más importante de todos, esto debido a su asociación con un peor pronóstico es la descendencia africana, según estudios realizados en Estado Unidos, el $40 \%$ de los casos reportados fueron en mujeres afroamericanas (2) 
Otro factor importante es la edad materna avanzada, esto debido a que, el $50 \%$ de los casos se presenta en mujeres mayores de 30 años, y en mujeres mayores de 40 años el riesgo de presentar la enfermedad es 20 veces mayor que en aquellas mujeres menores de 20 años (2,7). La patología se asocia con la multiparidad, así como en aquellas mujeres con embarazos múltiples, factor que fue documentado en hasta un $14.5 \%$ de los casos de cardiomiopatía periparto en Estado Unidos (2).

Los trastornos hipertensivos se presentan como otro factor de riesgo importante, en donde, la preeclampsia está presente en el $22 \%$ de las mujeres con cardiomiopatía periparto, además otros trastornos hipertensivos están presentes en un $37 \%$ de los casos (7).

\section{FISIOPATOLOGÍA}

En la actualidad la fisiopatología sigue siendo desconocida, aunque hay varios mecanismos que se han propuesto como los causantes de la enfermedad. Entre ellos se encuentra procesos autoinmunes, factores inflamatorios, infecciones agudas, malnutrición, alteraciones hormonales, apoptosis celular, disfunción y estrés oxidativo endotelial $(8,9)$.

Además, se ha asociado a una alteración en el metabolismo de la prolactina, la cual es una hormona hipofisiaria y de la forma soluble de la tirosina cinasa 1 (sFlt-1), la cual es una hormona placentaria (10).

En algunas mujeres se ha documentado un aumento de la expresión del gen 16kDa que es un fragmento de la prolactina que induce a que exista mayor estrés oxidativo, aumento de apoptosis y alteración en los mecanismos de hipoxia y oxigenación; lo que lleva a mayor daño miocárdico y síntomas de falla cardiaca (9).

También el aumento en la secreción placentaria de sFlt1 inhibe el factor de crecimiento endotelial vascular, agravando así el daño endotelial (8).

\section{GENÉTICA}

Es de gran importancia mencionar el rol de la genética en la fisiopatología de la cardiomiopatía periparto, esto debido a que, el modelo de fisiopatología hormonal/vascular descrito previamente no explica la razón de por qué solamente ciertas mujeres desarrollan la patología; por lo que, se han realizado estudios genéticos, donde se han documentado mutaciones genéticas dañinas en un $15-20 \%$ de las pacientes con cardiomiopatía periparto, $(6,8)$ siendo la más frecuente en el gen TTN, el cual se encarga de codificar la titina, la cual es la proteína humana más grande, y ejerce una función de gran importancia a nivel del musculo cardiaco, especialmente en el sarcómero, donde permite una adecuada recuperación del balance es la estructura del sarcomérica después de cada contracción $(5,7,10)$.

Este tipo de mutaciones también han sido documentadas en una proporción similar en paciente con cardiomiopatía dilatada idiopática, lo que indica que ambas comparten un trasfondo genético, sin embargo, no está claro el por qué algunas mujeres desarrollan cardiomiopatía periparto y otras desarrollan cardiomiopatía dilatada idiopática más adelante en la vida. Un $1 \%$ de la población general presentan mutaciones en el gen TTN sin enfermedad aparente (5) lo que habla del impacto de otros factores en el desarrollo de la enfermedad.

La presencia de mutaciones en el gen TTN, se correlaciona con fracciones de eyección menores al año de seguimiento, además estas mutaciones fueron encontradas en el $13 \%$ de mujeres con descendencia africana y solamente en el $8 \%$ de mujeres caucásicas, lo que podría explicar en parte el peor pronóstico que presentan en general las mujeres afrodescendientes $(7,11)$.

\section{CLÍNICA}


La mayoría presentan signos y síntomas de insuficiencia cardíaca congestiva (5). Los síntomas son muy variados, e incluyen fatiga, disnea de esfuerzo, disnea paroxística nocturna, tos, ortopnea, edema con fóvea en las extremidades inferiores, palpitaciones, dolor torácico, y dolor abdominal debido a la hepatomegalia $(1,4)$.

La exploración física suele revelar taquipnea, taquicardia, presión venosa yugular elevada, estertores pulmonares, ritmo de galope y ascitis. La presión arterial puede estar normal, reducida o elevada $(1,7)$.

También pueden tener taquiarritmias que incluyen taquicardia supra ventricular, fibrilación atrial y en casos muy raros taquicardia ventricular (12).

La evolución de la enfermedad varía mucho, y va desde una presentación leve y con menor frecuencia, pueden presentar shock cardiogénico que requiera soporte circulatorio inotrópico o mecánico $(8,11)$.

Los frecuentes retrasos en el diagnóstico se deben a la falta de reconocimiento de esta enfermedad y que los síntomas se pueden confundir con los cambios fisiológicos del embarazo y el periodo posparto $(2,13)$.

\section{DIAGNÓSTICO}

Antes de iniciar a describir la metodología diagnostica utilizada, es de gran importancia definir que la cardiomiopatía periparto permanece como un diagnóstico de exclusión $(7,14)$, se debe tener una amplia sospecha diagnostica en paciente al final del embarazo y en los primeros meses posparto, así como, un diagnostico diferencial completo, entre las patologías más importantes a descartar, se encuentran las siguientes: miocarditis, cardiomiopatía familiar idiopática preexistente, tromboembolismo pulmonar, embolismo de líquido, preeclampsia severa, enfermedad valvular cardiaca y cardiomiopatía hipertrófica; estas deben ser las patologías que debemos descartar previo a realizar un diagnóstico de cardiomiopatía periparto $(4,6)$.

En la evaluación de la cardiomiopatía periparto, esta suele presentarse en la mayoría de las mujeres en el periodo posparto, usualmente durante el primer mes después del parto, sin embargo, hay excepciones, las mujeres con descendencia africana se diagnostican con frecuencia más tarde en el periodo posparto (2).

En todo paciente con sospecha de cardiomiopatía periparto se debe obtener un ecocardiograma transtorácico tan pronto como sea posible, ya que es la forma más objetiva de documentar los cambios cardiacos sugestivos de la enfermedad, donde entre los criterios diagnósticos están los siguientes: fracción de eyección menor a $45 \%$, fracción de contracción miocárdica menor al $30 \%$ y dimensiones del ventrículo izquierdo al final de la diástole mayores a 2.7 $\mathrm{cm} / \mathrm{m} 2$, puede o no haber dilatación del ventrículo izquierdo $(7,15)$. El otro examen que complementa el diagnóstico es la realización de medición de péptidos natriuréticos (BNP y NT pro-BNP), ya que, estos durante el embarazo normal se encuentran elevados y están marcadamente elevados en presencia de la patología, por lo que, si bien no son específicos de la patología al valorarlo en conjunto con el ecocardiograma, ambas alteraciones son sugestivas de la patología (2).

Las otras dos pruebas que se realizan de manera complementaria son el electrocardiograma, el cual está alterado en el $90 \%$ de los pacientes (15), suele mostrar taquicardia sinusal, así como, cambios inespecíficos en la repolarización (4); y la radiografía de tórax, la cual suele documentar: cardiomegalia, congestión pulmonar y derramen pleural (4).

La resonancia magnética se podría utilizar en casos seleccionados para realizar una valoración adicional de la morfología y 
ayudar a prevenir resultados adversos, sin embargo, no hay un patrón especifico de la patología y no se usa de manera rutinaria. La biopsia endomiocárdica no forma parte del algoritmo diagnóstico y tampoco se utiliza de manera rutinaria $(7)$.

\section{Tratamiento}

El manejo es similar al de otras causas de insuficiencia cardíaca e incluye mantener una adecuada oxigenación, restricción de líquidos y sal, frenar la progresión de la disfunción del ventrículo izquierdo mediante vasodilatación y diuréticos y mejorar la supervivencia $(7,12)$.

$\mathrm{Si}$ la patología se presenta como insuficiencia cardiaca aguda, el tratamiento debe instaurarse de manera inmediata, e incluye oxígeno para mantener una saturación por encima del $95 \%$, además la administración de furosemida intravenosa para disminuir la congestión y sobrecarga de volumen. Si presenta una presión arterial sistólica mayor de $110 \mathrm{~mm} \mathrm{Hg}$ se pueden utilizar los nitratos intravenosos (16).

Cuando hay gasto cardiaco bajo, con síntomas y signos de hipoperfusión y se encuentran en shock cardiogénico, está indicado el uso de inotrópicos y en casos refractarios al tratamiento médico se pueden utilizar dispositivos de asistencia mecánica temporal como el balón intraaórtico y debe comenzarse un protocolo de trasplante cardiaco $(12,15,16)$.

En la insuficiencia cardíaca crónica el tratamiento farmacológico convencional incluye diuréticos, vasodilatadores, inotrópicos, Inhibidores de la enzima convertidora de angiotensina, inhibidores de los receptores II de angiotensina, beta bloqueadores, digoxina y espironolactona (9).

Los medicamentos inhibidores de la enzima convertidora de angiotensina II (IECA), los antagonistas del receptor de angiotensina II (ARA-II), son esenciales en el manejo de falla cardiaca, pero deben ser evitados durante el embarazo dado su efecto teratógeno. El tratamiento posparto con IECAS O ARA- II está indicado mientras el ventrículo izquierdo siga deteriorándose (16).

Se pueden utilizar como vasodilatadores de manera segura en el embarazo la hidralazina asociada a nitratos (nitroglicerina), estos reducen la precarga, la poscarga y la presión de llenado ventricular (12).

La digoxina, un agente inotrópico, mejora la sintomatología, calidad de vida, y tolerancia al ejercicio al aumentar la contractibilidad del miocardio, es seguro en el embarazo, se sugiere su uso en mujeres con disfunción ventricular izquierda sistólica y una fracción de eyección menor al $40 \%$ con signos y síntomas de insuficiencia cardíaca a pesar de estar en tratamiento $(7,9)$.

Los $\beta$ bloqueadores aprobados para el tratamiento de la insuficiencia cardíaca como metoprolol, carvedilol, y bisoprolol son seguros tanto en el embarazo como en el puerperio, se prefieren los bloqueadores $\beta 1$ selectivos que no inducen dinámica uterina. Estos mejoran la supervivencia y protegen contra las taquiarritmias $(9,12)$.

Los diuréticos se indican cuando hay evidencia clínica o radiológica de sobrecarga de volumen, y mejoran el edema pulmonar y periférico $(7,16)$.

Se prefieren los de asa como la furosemida. Pueden indicarse en el embarazo y puerperio, pero deben usarse con precaución para evitar hipotensión y disminución del flujo uteroplacentario $(9,17)$. Los antagonistas de la aldosterona, como la espironolactona, no se recomiendan durante el embarazo, por sus efectos anti androgénicos (17).

La bromocriptina inhibe la prolactina lo que sugiere un beneficio teórico del tratamiento, pero se requieren más estudios (7).

Por la influencia que genera la prolactina, se ha probado el buen efecto que tiene la 
bromocriptina, influenciado en disminución en las muertes, y en pacientes con clase funcional NYHA IIIIV y con fracción de eyección del ventrículo izquierdo

menor a $35 \%$, sin embargo, los estudios realizados son con poblaciones reducidas, por lo que se requiere más investigación (9). Las pacientes tienen alto riesgo de enfermedad tromboembólica, por lo que la anticoagulación profiláctica está indicada desde el diagnostico hasta que la fracción de eyección del ventrículo izquierdo sea mayor del 35\% $(12,16)$.

Se puede utilizar de manera segura las heparinas (no fraccionada y de bajo peso molecular) y está contraindicado el uso de warfarina durante el embarazo ya que es teratógeno (16).

El tratamiento no farmacológico consiste principalmente en la ingesta baja de sodio (menos de 2 g/día), restricción hídrica (menos $2 \mathrm{~L} /$ día), actividad física diaria si es tolerada y monitorización hemodinámica (9).

\section{PRONÓSTICO}

El pronóstico materno es variable, sin embargo, es mejor que para otras causas de cardiomiopatía, ya que, existe mejoría en la mayoría de los pacientes (7), aproximadamente el $50 \%$ de las pacientes presentan una mejoría en la fracción de eyección durante los primeros 6 meses posparto, donde recuperación se define como una fracción de eyección mayor o igual a $50 \%$ en este periodo $(5,13,18)$. Sin embargo, una recuperación retrasada también ha sido documentada, esto de manera frecuente en paciente con descendencia africana, la cual se asocia con diagnostico tardío, peor fracción de eyección al diagnóstico y mayor mortalidad (7).

La mortalidad general es de aproximadamente $4 \%$ (5), sin embargo, esto puede variar geográficamente como fue descrito previamente, con mortalidades mayores en aquellas pacientes con descendencia africana, las cuales, presentan 6.4 veces más riesgo de fallecer por complicaciones asociadas que las mujeres caucásicas; la mortalidad incrementa por otros factores, tales como: edad materna elevada y multiparidad y fracciones de eyección menores a $25 \%$ al momento del diagnóstico, con el $87 \%$ de las muertes ocurriendo en los primeros seis meses posteriores al diagnóstico (17).

El principal factor pronóstico de recuperación a largo plazo es la fracción de eyección al momento del diagnóstico, donde fracciones de eyección del ventrículo izquierdo menores a $30 \%$ se asociaron con tasas más bajas de recuperación y un riesgo mayor de efectos adversos; (2). Donde el tromboembolismo fue el más frecuente, un $10-17 \%$ de las pacientes presentaron un trombo en el ventrículo izquierdo al momento del diagnóstico inicial.

\section{Embarazos subsecuentes}

Un embarazo subsecuente en una mujer con historia de cardiomiopatía periparto, está asociado con riesgo de recurrencia, disfunción cardiaca persistente y mortalidad; donde el riesgo es substancialmente mayor en mujeres con disfunción persistente previo al nuevo embarazo (17).

Aquellas pacientes que presentan el menor riesgo de recurrencia son las que presentan fracciones de eyección mayores a $55 \%$, con adecuada reserva de contractilidad durante el ecocardiograma, y que la fracción de eyección no disminuya al retirar medicamentos tales como: IECA o ARA2. Tanto las pacientes con adecuada fracción de eyección como aquellas con fracción persistentemente disminuida demostraron una reducción en la fracción de eyección (FE) con el embarazo subsecuente; sin embargo, aquellas con FE mayor a $55 \%$ tienen una mortalidad cercana al $0 \%$ y aquellas con $\mathrm{FE}$ disminuida una mortalidad de 19\%; por lo que aquellas con FE recuperada pueden seguir adelante con el 
embarazo bajo la atención de un equipo multidisciplinario (14).

Aquellas mujeres con FE disminuida de manera persistente deben ser aconsejadas sobre los riesgos de un embarazo subsecuente, y recomendar métodos anticonceptivos de larga duración (15). Debido al alto riesgo tromboembólico que presentan las mujeres con cardiomiopatía periparto y FE disminuida se deben evitar todos los anticonceptivos que presenten estrógenos en su formulación; dejando como primera línea los dispositivos liberadores de progesterona, tanto el dispositivo intrauterino, como el implante subdérmico, la ligación tubárica también es una opción viable (15).

Un embarazo posterior sin complicaciones no puede predecir el riesgo de embarazos posteriores, de igual manera, el principal factor pronostico va ser la FE previo al embarazo (15).

\section{CONCLUSIONES}

La cardiomiopatía periparto es una enfermedad poco frecuente, con una incidencia en aumento, y una fisiopatología desconocida, la cual siempre se debe considerar en el diagnóstico diferencial de mujeres al final del embarazo o en el posparto, con síntomas de insuficiencia cardíaca congestiva, ya que los retrasos en el diagnostico ocasionan un aumento en la mortalidad y complicaciones severas que se pudieron evitar al realizar un diagnóstico oportuno.

Es de suma importancia conocer las bases del manejo de esta patología para poder actuar de manera correcta una vez diagnosticada, la ventaja es que no difiere no del tratamiento utilizado para otras causas de falla cardíaca, ya que con un adecuado manejo la mayoría de las pacientes se recuperan y se evitan complicaciones mayores.

\section{Los autores declaran no tener conflicto de interés.}

\section{REFERENCIAS}

1. Azibani F, Sliwa K. Peripartum Cardiomyopathy: An Update. Curr Heart Fail Rep. octubre de 2018;15(5):297-306.

2. Davis MB, Arany Z, McNamara DM, Goland S, Elkayam U. Peripartum Cardiomyopathy. J Am Coll Cardiol. enero de 2020;75(2):207-21.

3. Asad ZUA, Maiwand M, Farah F, Dasari TW. Peripartum cardiomyopathy: A systematic review of the literature. Clin Cardiol. mayo de 2018;41(5):693-7.

4. Cunningham FG, Byrne JJ, Nelson DB. Peripartum Cardiomyopathy. Obstet Gynecol. enero de 2019;133(1):167-79.

5. Arany Z. Understanding Peripartum Cardiomyopathy. 2017;12.

6. Bauersachs J, König T, Meer P, Petrie MC, Hilfiker-Kleiner D, Mbakwem A, et al. Pathophysiology, diagnosis and management of peripartum cardiomyopathy: a position statement from the Heart Failure Association of the European Society of Cardiology Study Group on peripartum cardiomyopathy. Eur J Heart Fail. julio de 2019;21(7):827-43.

7. Cruz MO, Briller J, Hibbard JU. New Insights in Peripartum Cardiomyopathy. Obstet Gynecol Clin North Am. junio de 2018;45(2):281-98.

8. Koenig $\mathrm{T}$, Hilfiker-Kleiner $\mathrm{D}$, Bauersachs $\mathrm{J}$. Peripartum cardiomyopathy. Herz. agosto de 2018;43(5):431-7.

9. Durán-Morales MA, Ariza-Parra EJ. Miocardiopatía periparto: patología potencialmente mortal. :9.

10. Auger N, Ukah UV, Potter BJ. Peripartum cardiomyopathy: a family affair? Heart. julio de 2019;105(14):1051-2.

11. Honigberg MC, Givertz MM. Peripartum cardiomyopathy. BMJ. 30 de enero de 2019;k5287.

12. Thompson L, Hartsilver E. Peripartum cardiomyopathy. 2016;31:4.

13. Jagodzińska A, Gondek A, Pietrzak B, CudnochJędrzejewska $A$, Mamcarz $A$, Wielgoś $M$. Peripartum cardiomyopathy - from pathogenesis to treatment. :9.

14. Baris L, Cornette J, Johnson MR, Sliwa K, RoosHesselink JW. Peripartum cardiomyopathy: disease or syndrome? :6.

15. Zagelbaum NK, Bhinder J, Gupta CA, Frishman WH, Aronow WS. Peripartum Cardiomyopathy Incidence, Risk Factors, Diagnostic Criteria, Pathophysiology, and Treatment Options. Cardiol Rev. mayo de 2020;28(3):148-55. 
16. Torga JEP, Rubio PAR, García I, Ramírez NMS, Arocha IC, Boffil P. Peripartum Cardiomyopathy. $: 15$.

17. Goland S, Elkayam U. Peripartum cardiomyopathy: approach to management. 2018;33(00):7.

18. Gupta D, Wenger NK. Peripartum cardiomyopathy: Status 2018. Clin Cardiol. febrero de 2018;41(2):217-9. 